\title{
Meeting Report
}

\section{Redox regulation by ischemia/reperfusion}

\author{
M Maccarrone ${ }^{*, 1}$ and B Brüne ${ }^{\star, 2}$ \\ ${ }^{1}$ Department of Biomedical Sciences, Faculty of Veterinary Medicine, University of Teramo, Teramo, Italy \\ 2 Institute of Biochemistry I, Faculty of Medicine, Johann Wolfgang Goethe-University, Frankfurt, Germany \\ * Corresponding authors: M Maccarrone, Department of Biomedical Sciences, University of Teramo, Piazza A Moro 45, I-64100 Teramo, Italy. \\ E-mail: mmaccarrone@unite.it or B Brüne, Institute of Biochemistry I, Faculty of Medicine, Johann Wolfgang Goethe-University, 60590 Frankfurt, Germany. \\ E-mail: bruene@zbc.kgu.de
}

Cell Death and Differentiation (2006) 13, 2002-2003. doi:10.1038/sj.cdd.4401971; published online 2 June 2006

Villa Vigoni Conference 'Redox regulation by ischemia/reperfusion', Villa Vigoni, Loveno di Menaggio, Italy, 29 March-1 April 2006

In the past years, the Italian-German Villa Vigoni conferences have represented a forum discussing redox-regulated reactions that affect human health. The impact of redox regulation by ischemia/reperfusion has been the focus of the ninth conference, held at Loveno di Menaggio, Italy. The fascinating architecture of Villa Vigoni that faces the beautiful Lago di Como in northern Italy, a place known for its tradition in art and science, created an excellent environment to reach out for something vague, known as 'redox regulation'.

As molecular oxygen is a terminal electron acceptor during oxidative phosphorylation, partial reaction of oxygen with electrons provokes the formation of a variety of reactive oxygen species (ROS), either used for cell signaling or, if the antioxidant capacity is exhausted, for extensive cell damage. Therefore, reduction of $\mathrm{O}_{2}$ is tightly regulated and sources of oxidative stress are under the control of a multifaceted antioxidant defence. In addition, reactive nitrogen species (RNS) have long been implicated as damaging biomolecules, which are considered fundamentally pathogenic in diverse diseases. It is now recognized that redox alterations are not solely mediated through gross damage but also by their discriminating role as redox regulators of cellular signaling. The redox regulators superoxide $\left(\mathrm{O}_{2}^{-}\right)$or nitric oxide (NO) are generated by locally and mechanistically defined reactions, which differ according to the physiological stimulus or the pathophysiological condition. The term 'redox signaling' describes the regulation of enzymes and mediators by oxidation and subsequent reduction, as part of a signal-transducing network governed by the levels of the pro-oxidant molecules and antioxidant fluxes. However, it is not only the metabolism towards ROS or RNS that accounts for redox regulation, but rather the $\mathrm{O}_{2}$ availability itself contributes in multiple ways. The ability to sense and respond to changes in oxygenation represents a fundamental property to assure the cellular $\mathrm{O}_{2}$ supply to be within a narrow range, which balances the risks of oxidative damage versus oxygen deficiency. A number of issues on redox regulation by ischemia/reperfusion were discussed at the 2006 Villa Vigoni conference.

\section{Inflammatory genes, mitochondria and oxygen sensing}

First, the role of the endogenous tone of ROS and RNS with concomitant formation of peroxynitrite, was reviewed. Interestingly, $\mathrm{ONOO}^{-}$provokes cell survival in inflammatory cells such as monocytes/macrophages by initiating multiple pathways that culminate in phosphorylation of $\mathrm{Bad}$. $\mathrm{NO} / \mathrm{O}_{2}^{-}-$ evoked stress was put in the context of antioxidant systems such as thioredoxins, peroxiredoxins and glutathione peroxidases, with implications for outside-in signaling, parasite growth and gene activation during cancer. We addressed the role of $\mathrm{NO}$ in mitochondrial biogenesis related to caloric restriction and human obesity and focused on cyclophilin $D$ in modulating sensitivity of mitochondrial permeability transition. Thereafter, the tempting hypothesis was introduced that morphine and related compounds stimulate NO production, thereby depressing mitochondrial fluorescence to mediate the antinociceptive action of these agents.

Oxygen sensing was a further priority theme. A way to visualize hypoxia-mediated signaling pathways was presented by using fluorescence recovery after photobleaching (FRAP) and fluorescence resonance energy transfer (FRET), and was followed by considerations to explain HIF- $1 \alpha$ versus $-2 \alpha$ in evoking distinct but also overlapping gene profiles, depending on the cell type and cell culture conditions. The impact of NO in attenuating accumulation of $\mathrm{HIF}-1 \alpha$ under hypoxia was then associated with impaired oxygen consumption, thus leaving more oxygen available for enzymatic destruction of HIF- $1 \alpha$ via prolyl hydroxylation. Alternatively, hypoxia/NO might activate the calpain system to account for a so far unappreciated destruction of HIF-1 $\alpha$, irrespective of the proteasome.

\section{Ischemia/reperfusion in the periphery and central nervous system}

This session addressed the issue of cell death induced in the heart by ischemia/reperfusion injury as well as cardioprotective effect of urocortins, which engage multiple-signaling 
pathways such as MAPK, STAT-3 and mitochondrial $\mathrm{K}_{\text {ATP }}$ channels. Ischemic injury of the gut was discussed with respect to ROS formation and poly(ADP-ribose) polymerase (PARP) activation, whereas the focus was on NF- $\kappa \mathrm{B}$ and NO with a likely feedback on cellular rescue systems during ischemic insults of pancreatic cancer cells. During cold ischemia, in hepatocytes substances able to mimic catalase and chelate iron were protective with the notion that chelatable iron in mitochondria increase in cold-injured cells. In hepatic ischemia/reperfusion injury, heme oxygenase catalyzes the conversion of heme to bilirubin/biliverdin, and concomitantly releases $\mathrm{CO}$, which protects hepatic microcirculatory dysfunction after hemorrhagic shock. Central implications of ischemia/ reperfusion were discussed with respect to NF- $\kappa \mathrm{B}$ signaling. In silico promoter analysis predicts activation of $\mathrm{NF}-\kappa \mathrm{B}$, which contributes to inflammation and apoptosis during middle cerebral artery occlusion (MCAo). Within the striatum, a fall in ATP was shown to be critical, with medium spiny neurons being most sensitive to ischemia. The data allowed to define a 'post-ischemic long-term potentiation' (i-LTP) process, which is dangerous for cells and is different from the physiological LTP, responsible for learning and memory. Moreover, in the ischemic core (striatum), but not in the penumbra (cortex), of rats subjected to transient MCAo, endogenous levels of anandamide increased owing to reduced degradation and increased synthesis, thus contributing to plastic changes in models of ischemia/reperfusion.

\section{Concluding remarks and future perspectives}

A concept was proposed to understand complex signal transmission under conditions of ischemia/reperfusion. Responses are determined by superoxide-initiated signaling (oxysome), NO-evoked responses (nitrosome) and hypoxiadriven alterations (hypoxysome) with a general consensus that mitochondria are a converging point of different redoxregulated pathways. It was highlighted that future research should focus on the time course of the shift from aerobic to anaerobic metabolism, which supposedly represents a crucial check-point of redox regulation by ischemia/reperfusion. It is believed that human pathologies such as the metabolic syndrome or acute versus chronic inflammation are crucially affected by redox regulation, and thus may be in the focus of an upcoming Villa Vigoni conference.

\section{Acknowledgements}

We thank all participants for their valuable contributions. Financial support by Facoltà di Medicina Veterinaria, Dipartimento di Scienze Biomediche Comparate and Dipartimento di Scienze Cliniche Veterinarie of the Università degli Studi di Teramo (to $\mathrm{MM}$ ) and by Deutsche Forschungsgemeinschaft (to German participants) is gratefully acknowledged. 